\title{
Ein Theorie- und Praxismanual für wen?
}

Das Ihnen vorliegende Theorie- und Praxismanual wurde für alle mit Stress und Entspannung befassten Praxisbereiche, insbesondere aber für die folgenden Berufsgruppen entwickelt:

- Ärzte

- Psychotherapeuten

- Psychologen

- Pädagogen

- Sozialpädagogen

- Sozialwissenschaftler

- Gesundheitswissenschaftler

- Sportwissenschaftler

- Diplom-Sportlehrer

- Sport- und Gymnastiklehrer

- Erzieher

Das Manual eignet sich sowohl für die Aus- als auch für die (eigene) Fort- bzw. Weiterbildung in diesen unterschiedlichen Praxisbereichen.

Von ganz wesentlicher Bedeutung ist es für die einzelnen Berufsgruppen dabei zu erkennen, worin die eigenen Möglichkeiten und Grenzen im Umgang mit dem Phänomen Stress und den unter Stress leidenden Patienten bzw. Klienten bestehen.

Wie Sie auf den folgenden Seiten sehen werden, erfordert das Phänomen Stress sehr unterschiedliche Betrachtungs- und Herangehensweisen. Hierfür ist es zunächst erforderlich, dass die überwiegend mit Stress befassten Berufsgruppen Grundlagenkenntnisse im Umgang mit der Thematik erwerben. 